\title{
Intergalactic HII regions in Stephan's Quintet
}

\author{
C. Mendes de Oliveira $^{1} \dagger$, E. S. Cypriano ${ }^{1}$, \\ L. Sodré Jr. ${ }^{1}$ and C. Balkowski ${ }^{2}$ \\ ${ }^{1}$ Instituto de Astronomia, Geofísica e Ciências Atmosféricas, Departamento de Astronomia, \\ Universidade de São Paulo, Brazil email: oliveira@astro.iag.usp.br \\ ${ }^{2}$ Observatoire de Paris, GEPI, CNRS and Universite Paris 7, 5 Place Jules Janssen, F-92195 \\ Meudon Cedex, France
}

\begin{abstract}
We have discovered four intergalactic HII regions in the Stephan's Quintet, at more than $25 \mathrm{kpc}$ projected distance from the center of the nearest group galaxy, with no apparent optical connection to it. They have $\mathrm{M}_{B}$ ranging from -11.9 to $-12.5 \mathrm{mag}$, colors $\mathrm{B}-\mathrm{R}=0.7$ to $1.1 \mathrm{mag}$, radial velocities from 6565 to $6651 \mathrm{~km} / \mathrm{s}$ and they are superposed onto the HI tail east of NGC 7319, with a mean radial velocity of $6610 \mathrm{~km} / \mathrm{s}$. In addition, they have metalicities of the order of $12+\log (\mathrm{O} / \mathrm{H})=8.58 \pm 0.25$, which suggests that they were formed from preenriched material. We derive a mean age of $4.6 \pm 0.6 \mathrm{Myr}$ and a mean stellar mass of $(2.9 \pm$ $1.4) \times 10^{4} M_{\odot}$ for the four objects. The masses, ages, colors, velocities, metalicities and location of the objects suggest that they are HII regions that were formed far away from the galaxies through compression of the intergalactic HI gas by galaxy collisions.
\end{abstract}

\section{Introduction}

About a decade ago the first convincing examples of new stellar systems formed during galaxy collisions were established. In the high-end of the mass spectrum, the tidal dwarf galaxies were shown to be formed at the ends of tidal tails. In the lower-end of the mass spectrum, young star clusters, possible proto-globular clusters, were identified in gas-rich mergers. The formation of tidal dwarf galaxies and young clusters during galaxy collisions is nowadays an active topic of study. Besides helping in exploring the connection to the host galaxy, the properties of these stellar systems aid at understanding the poorly known processes of star formation during interaction events.

A natural environment where to look for new objects formed in galaxy collisions is in interacting compact groups, small groups of galaxies with large spatial densities, low velocity dispersions, and a high rate of interactions. In this paper, we report on the discovery of another class of objects (different from the tidal dwarfs and the young clusters) which may be formed as a consequence of galaxy collisions: intergalactic HII regions in the Stephan's Quintet.

\section{Observations}

A 15-min r' image of the quintet (shown in Fig. 1), a 22-min b' image and a 15-min multi-slit exposure (covering from 4000 to $8000 \AA$, with a resolution of $8 \AA$ ) were obtained with GMOS on the Gemini North Telescope. The spectra of the four intergalactic HII regions (IHII) are shown in Fig. 1b. As can be seen, these are typical of HII regions.

$\dagger$ Present address: Universitäts-Sternwarte der Ludwig-Maximilians-Universität, Scheinerstrasse 1, D-81679 München, Germany 
This, together with the large projected distance to the closest bright member galaxy $(>25 \mathrm{kpc})$, justifies the name intergalactic HII regions, IHII.

\section{Results and discussion}

Radial velocities: The heliocentric velocities of the IHII regions derived from the redshifts of the brightest lines are indicated in Fig. 1a. From Fig. 7 of Williams et al. (2002), we note that the velocities of all IHII's coincide with the HI velocities from the channel maps within $\pm 20 \mathrm{~km} \mathrm{~s}^{-1}$, suggesting a physical association of the knots with the HI cloud.

Metalicities: In order to estimate the metalicities of the objects, we have adopted the N2 calibrator, proposed by Denicoló et al. (2002), which is defined as the logarithm of the $[\mathrm{NII}] \lambda 6584 / \mathrm{H} \alpha$ ratio. The metalicities inferred for the four objects are very similar, the mean value being $12+\log (\mathrm{O} / \mathrm{H})=8.58$. This value is close to solar: $12+\log (\mathrm{O} / \mathrm{H}) \odot$ $=8.8 \pm 0.1$, (Grevesse \& Sauval 1998), suggesting that the IHII's have been formed from pre-enriched material.

Star burst parameters: We assume that the IHII are excited by young stars formed in an instantaneous star burst and use Starburst99 (Leitherer et al. 1999), with solar metalicity and Salpeter IMF, to estimate their physical parameters. From a comparison between the observed $\mathrm{H} \alpha$ equivalent widths and those produced by Starburst99, we find ages ranging from 3.2 to 5.6 Myr, with a mean age of $4.6 \pm 0.6 \mathrm{Myr}$ for the four regions. The mean mass is $(2.9 \pm 1.4) \times 10^{4} M_{\odot}$.

The IHII's found in Stephan's Quintet present the following characteristics: (1) they are compact (except for region b), (2) relatively high metallicities, (3) masses of the order of $10^{4} M_{\odot}$, (4) ages ranging from 3.2 to $5.6 \mathrm{Myr}$, (5) far away from the bright galaxies and with no apparent optical connection to them, (6) they coincide in location and velocity with the HI tail detected 2 arcmin east of NGC 7319 (Williams et al. 2002). The extremely young ages of these objects suggest that they, most probably, are located where they were born. We favour a scenario where the IHII's were formed in the intragroup medium, within the HI tail, through compression of the intergalactic HI gas by galaxy collisions. Further study of these objects is relevant since IHII and intragroup cluster formation might have been more important at higher redshifts, when interactions and mergers happened more frequently. A paper describing the details of this work can be found in Mendes de Oliveira et al. (2004). Similar objects have been recently found in several other systems by Ryan-Weber et al. (2004).

\section{Acknowledgements}

The authors would like to thank the Brazilian FAPESP, the PICS program, the Brazilian PRONEX program and the CNPq. CMdO deeply acknowledges the funding from the Alexander von Humboldt Foundation.

\section{Bibliography}

Denicoló, G., Terlevich, R. \& Terlevich, E. 2002, MNRAS, 330, 69

Leitherer, C., et al., 1999, ApJS, 123, 3

Mendes de Oliveira, C., Cypriano, E.S., Sodré Jr., L., Balkowski, C., 2004, ApJ, 605, 17 Ryan-Weber et al. 2004, AJ, 127, 1431

Williams, B.A., Yun, M.S., Verdes-Montenegro, L., 2002, AJ, 123, 241 

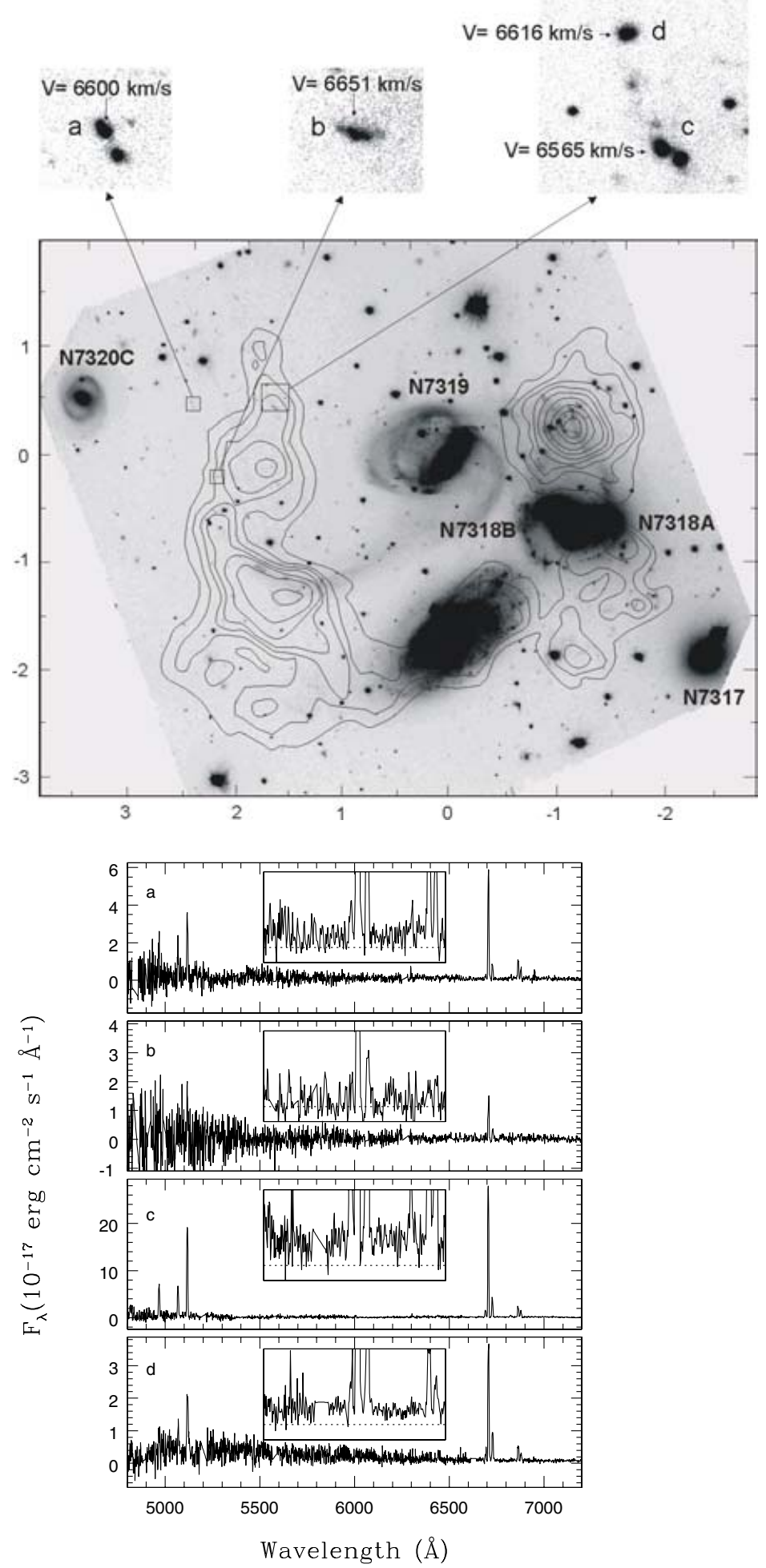

Figure 1. Top - An r' image of the Stephan's Quintet with the HI contours from Williams et al. (2002) overplotted. The contours are 5.8, 15, 23, 32, 44, 61, 87, 120, $180 \times 10^{19}$ atoms cm $^{-2}$. The three boxes in the upper left corner (and corresponding zoomed images) indicate the new IHII's. We note that all regions are within the HI tail. Region "a" coincides with the lower-level contour of $1.5 \times 10^{19}$ atoms $\mathrm{cm}^{-2}$ (not plotted). Bottom - Spectra of IHII "a" to "d". Each inset presents a zoom into the region from 6500 to $6900 \AA$. Zero flux is indicated with a dashed

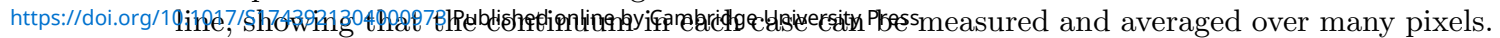

